\title{
COMPORTAMENTO DE SUPERFOSFATO SIMPLES CONTENDO FOSFATO DE FERRO DE BAIXA SOLUBILIDADE EM ÁGUA EM SOLOS DE VÁRZEA DO RIO GRANDE DO SUL ${ }^{(1)}$
}

\author{
Gerônimo Rodrigues Prado(2), Leandro Souza da Silva ${ }^{(3)}$, Luis \\ Ignácio Prochnow $^{(4)}$, Gustavo Griebeler $^{(5)}$, Elisandra Pocojeski ${ }^{(5)} \&$ \\ Vagner João Moro ${ }^{(6)}$
}

\begin{abstract}
RESUMO
Algumas rochas utilizadas na produção de fertilizantes contêm impurezas de baixa solubilidade em água, destacando-se alguns fosfatos de $\mathrm{Fe}$ e $\mathrm{Al}$, que poderiam ser aplicados em solos alagados, onde as reações de redução podem aumentar a solubilidade. $O$ objetivo deste trabalho foi estudar a liberação do $P$ de fertilizantes contendo impurezas de fosfatos de Fe com baixa solubilidade em água em diferentes solos de várzea do Rio Grande do Sul (RS) sob condições de alagamento. Para realização do experimento foram utilizados seis solos de várzea (dois Gleisssolos, dois Planossolos e dois Chernossolos) de diferentes locais do Estado do Rio Grande do Sul, os quais foram acondicionados em vasos plásticos de $8 \mathrm{~L}$. Cada solo recebeu duas fontes de $\mathbf{P}$ (superfosfato simples e superfosfato simples com resíduos de $\mathrm{Fe}$ ) e uma testemunha sem adição de $P$, com três repetições. Nos tratamentos com $P$ foram adicionados ao solo $100 \mathrm{mg} \mathrm{kg}^{-1} \mathrm{P}$ no solo. Os solos foram alagados e mantidos, em delineamento inteiramente casualizado e três repetições, com uma lâmina de água de $5 \mathrm{~cm}$ de altura acima de sua superfície. A solução do solo foi coletada semanalmente, sendo medidos imediatamente o $\mathrm{pH}$ e o potencial redox (Eh); posteriormente, procedeu-se, em laboratório, à determinação dos teores de $\mathrm{P} \mathrm{e} \mathrm{Fe}$. Os resultados mostram redução do Eh e aumento do pH e dos teores de $\mathrm{Fe}$ e $\mathrm{P}$ em
\end{abstract}

\footnotetext{
(1) Parte da Dissertação de Mestrado do primeiro autor apresentada no Programa de Pós-Graduação em Ciência do Solo da Universidade Federal de Santa Maria - UFSM. Recebido para publicação em abril de 2010 e aprovado em janeiro de 2011.

(2) Biólogo, Mestre em Ciência do Solo. Aluno do PPGMAA, Universidade Federal do Rio Grande do Sul - UFRGS. Rua Sarmento Leite 500, ICBS Laboratório de Micologia Ambiental, sala 209, CEP 90050-170 Porto Alegre (RS). E-mail: geronimo.prado@yahoo.com.br

(3) Professor do Departamento de Solos da Universidade Federal de Santa Maria - UFSM. Campus Universitário, CEP 97105-900 Santa Maria (RS). E-mail: leandro@smail.ufsm.br

(4) Engenheiro-Agrônomo, Diretor do Programa International Plant Nutrition Institute no Brasil (IPNI). Rua Alfredo Guedes 1949, Sala 701, CEP 13.416-901 Piracicaba (SP). E-mail: lprochnow@ipni.net

(5) Engenheiro-Agrônomo, aluno do PPGCS, UFSM. E-mails: gustavogriebeler@hotmail.com; epocojeski@gmail.com

(6) Engenheiro-Agrônomo, aluno do PPGCS, UFSM. E-mail: vagnermoro@yahoo.com.br
} 
solução para todos os solos. O teor de $\mathrm{P}$ na solução não diferiu quanto à fonte, mostrando que o superfosfato simples com impurezas de fosfato de Fe tem potencial de uso em solos de várzea do Rio Grande do Sul cultivados com arroz irrigado por alagamento, mesmo sem a solubilidade em água prevista na legislação brasileira.

Termos de indexação: fertilizantes, fósforo, impurezas, arroz irrigado.

\title{
SUMMARY: BEHAVIOR OF SIMPLE SUPERPHOSPHATE CONTAINING IRON PHOSPHATE WITH LOW WATER SOLUBILITY IN FLOODED LOWLAND SOILS IN THE STATE OF RIO GRANDE DO SUL
}

\begin{abstract}
Some rocks used in fertilizer production contain low water-soluble impurities, such as iron and aluminum phosphates. The industrialization processes of these rocks may generate simple superphosphate with an insufficient solubility degree to meet the requirements established by Brazilian fertilizer law. If applied to waterlogged soils, reduction reactions can increase the solubility of such fertilizers. The objective of this study was to evaluate phosphorus release from these fertilizers from flooded lowland soils of Rio Grande do Sul State. Six lowland samples were collected: two Albaqualf (Santa Maria and Pelotas); two Alfissol (Santa Maria and Cachoeirinha) and two Molisol (Santa Vitória do Palmar and Uruguaiana). Each soil received two phosphate sources - simple superphosphate and superphosphate with iron phosphate impurities - and a treatment without phosphorus addition. In the treatments with phosphorus, an amount equivalent to $100 \mathrm{mg} \mathrm{kg}^{-1} \mathrm{P}$ was applied. Soils in $8 \mathrm{~L}$ plastic buckets were flooded and a $5 \mathrm{~cm}$ water layer was maintained over the soil surface, in a complete randomized design with three replications. Soil solution was collected weekly and, immediately, the $\mathrm{pH}$ and redox potential (Eh) measured; afterwards, $P$ and $F e$ were extracted in the lab. Results show that Eh decreased and $\mathrm{pH}, \mathrm{P}$ and Fe content increased in the soil solution in all tested soils. The P increase in soil solution was independent of the phosphate source, indicating simple superphosphate with iron phosphate impurities for use in lowland soils where flooded rice is grown in Rio Grande do Sul, even though it does not comply with the solubility requirements established by the Brazilian fertilizer legislation.
\end{abstract}

Index terms: Fertilizers, phosphorous, impurity, flooded rice.

\section{INTRODUÇÃO}

As principais fontes industriais de fertilizantes contendo P utilizadas na agricultura são obtidas por meio do tratamento de rochas fosfáticas (fosfatos naturais). Em razão da baixa solubilidade em água, os fosfatos naturais podem sofrer processos físicos (lavagem e, ou, flotação) e químicos (acidulação) com ácido sulfúrico $\left(\mathrm{H}_{2} \mathrm{SO}_{4}\right)$ e ácido fosfórico $\left(\mathrm{H}_{3} \mathrm{PO}_{4}\right)$. O tratamento visa aumentar a solubilidade do fertilizante em água e a concentração de $\mathrm{P}$, produzindo o superfosfato simples, o triplo e outros fertilizantes fosfatados, comumente denominados de fertilizantes acidulados ou solúveis (Kaminski \& Peruzzo, 1997). O grau de solubilidade em água é importante e a legislação brasileira estabelece teores mínimos de $\mathrm{P}$ solúvel em água em 15, 36, 38 e $44 \%$, respectivamente, para o superfosfato simples, superfosfato triplo, DAP e MAP (Brasil, 2004).

Durante os processos de beneficiamento e acidulação dos fosfatos de rocha, quantidades significativas de compostos contendo fosfato de $\mathrm{Fe}$ (genericamente denominados de compostos tipo P-Fe) são descartadas no sentido de produzir fertilizantes que atendam às especificações da legislação quanto ao grau de solubilidade em água (Prochnow, 2001). Dessa maneira, quando concentrados apatíticos com muitas impurezas catiônicas ( $\mathrm{Fe}$ e $\mathrm{Al}$ ) sofrem acidulação, compostos contendo em sua composição $\mathrm{Fe}$ e $\mathrm{Al}$ (P-Fe ou $\mathrm{P}-\mathrm{Al}$ ) insolúveis em água - podendo ou não ser solúveis em citrato de amônio mais água $\left(\mathrm{CNA}+\mathrm{H}_{2} \mathrm{O}\right)$ - descaracterizam a qualidade do fertilizante. Tendo em vista a escassez de fosfatos naturais de alta qualidade para a produção de fertilizantes acidulados, estudos têm sido desenvolvidos para o aproveitamento de rochas fosfáticas com maiores teores de impurezas (Prochnow, 2001; Prochnow et al., 2003, 2006).

O uso de fertilizantes com teores altos de impurezas provou ser eficiente em solos alagados cultivados com arroz irrigado (Prochnow, 2001). Solos alagados distinguem-se física, química e biologicamente dos solos de sequeiro. A diminuição do potencial redox (Eh) e o aumento do $\mathrm{pH}$ do meio até próximo à neutralidade em função das reações de oxirredução são algumas alterações químicas que ocorrem após o alagamento desses solos (Ponnanperuma, 1972; Vahl, 2004; Sousa et al., 2006). As alterações decorrentes 
do alagamento dos solos também modificam a disponibilidade e a dinâmica dos nutrientes nesses ambientes (Silva et al., 2003). O P está entre os nutrientes que sofrem alterações nas primeiras semanas de alagamento, aumentando sua concentração (Velloso et al., 1993). No solo, o P não está diretamente envolvido nas reações de oxidação e redução. No entanto, segundo Ranno (2004) e Ranno et al. (2007), os compostos P-Fe nos solos de várzea cultivados com arroz irrigado apresentam a maior contribuição para o $\mathrm{P}$ disponível às plantas, antes $\mathrm{e}$ após o alagamento. Isso ocorre pelo fato de o Fe (III), especialmente dos óxidos, ser utilizado como aceptor de elétrons na decomposição anaeróbia, provocando a solubilização do $\mathrm{P}$ adsorvido. Assim, parte-se do pressuposto de que o fenômeno da redução provocado pelo alagamento também irá solubilizar compostos PFe no fertilizante, mesmo que originalmente insolúveis em água.

A eficiência agronômica de três superfosfatos simples $\left(\mathrm{SSP}_{1}, \mathrm{SSP}_{2}\right.$ e $\left.\mathrm{SSP}_{3}\right)$, contendo diferentes graus de impurezas tipo $\mathrm{Fe}_{3} \mathrm{H}_{9}\left(\mathrm{PO}_{4}\right)_{6} \cdot 6 \mathrm{H}_{2} \mathrm{O}$, foi testada por Prochnow et al. (2003) em solos alagados cultivados com arroz. A análise da produção de matéria seca produzida evidenciou que o fertilizante com maior quantidade de impurezas de $\mathrm{P}-\mathrm{Fe}\left(\mathrm{SSP}_{3}\right)$ foi semelhante ao das demais fontes, inclusive quando comparado com a testemunha contendo todo o $\mathrm{P}$ solúvel em água. Resultados como esse mostram que fertilizantes com impurezas do tipo P-Fe insolúveis em água podem suprir as necessidades de $\mathrm{P}$ às plantas em condições de alagamento. Entretanto, alguns fatores, como a capacidade de fixação de $\mathrm{P}$ do solo e o curso de redução dos solos alagados, podem alterar a eficiência desses fertilizantes para o arroz irrigado por alagamento. Segundo Braga (2006), fontes de P com menor solubilidade em água podem apresentar maior eficiência agronômica relativa em solos de elevada capacidade de fixação de P. Os solos de várzea utilizados para cultivo do arroz são bastante variáveis em termos de características que interferem na dinâmica do $\mathrm{P}$, como $\mathrm{pH}$, teor de matéria orgânica $\mathrm{e}$ óxidos de Fe, área superficial específica dos minerais que compõem o solo, teor de Al trocável, entre outros
(Brady \& Weil, 2002). Alguns solos do Rio Grande do Sul apresentam diferentes capacidades de adsorção de $\mathrm{P}$, cujos valores variam de 71 a $933 \mathrm{mg} \mathrm{kg}^{-1}$, refletindo na quantidade de P necessária para alterar o P disponível do solo (Ranno et al., 2007). Assim, a liberação do $\mathrm{P}$ de compostos $\mathrm{P}$-Fe de fertilizantes pode ser alterada pela composição do solo e pelo curso de redução após o alagamento deste.

O objetivo deste trabalho foi estudar o comportamento do superfosfato simples contendo impurezas de fosfato de Fe de baixa solubilidade em água submetido a condições de alagamento em diferentes solos de várzea, comparando-o ao comportamento do superfosfato simples sem resíduos de fosfato de Fe.

\section{MATERIAL E MÉTODOS}

O trabalho foi realizado na casa de vegetação do Departamento de Solos da Universidade Federal de Santa Maria (UFSM), localizado no município de Santa Maria, no Estado do Rio Grande do Sul (RS). Amostras de seis solos (36 kg por solo) de várzea representativos da área de arroz irrigado no Estado do RS foram coletadas na camada de $0-20 \mathrm{~cm}$. Os solos coletados foram: dois Planossolos, um do município de Santa Maria, coletado na área experimental do Departamento de Solos da UFSM, e outro do município de Pelotas, coletado na área experimental da EMBRAPA/CPACT; dois Gleissolos, um do município de Santa Maria, coletado na área experimental do Departamento de Solos da UFSM, e outro do município de Cachoeirinha, coletado na área experimental do IRGA; e dois Chernossolos, um do município de Uruguaiana, coletado em propriedade rural, e outro do município de Santa Vitória do Palmar, coletado na área experimental da subestação do IRGA. Os solos foram secos ao ar, moídos e passados em peneira de $4 \mathrm{~mm}$. Após o peneiramento foi realizada análise química e física de cada solo (Quadro 1), de acordo com métodos descritos por Tedesco et al. (1995).

Quadro 1. Teor de argila e algumas características químicas dos solos estudados

\begin{tabular}{|c|c|c|c|c|c|c|c|c|c|c|c|}
\hline Solo & $\mathbf{P}$ & $\mathbf{K}$ & Arg & MO & $\mathrm{Al}^{3+}$ & $\mathrm{Ca}^{2+}$ & $\mathbf{H}+\mathbf{A l}$ & $\mathrm{CTC}_{\mathrm{pH}} 7$ & $F e_{o}^{(2)}$ & $F e_{d}^{(2)}$ & $\mathrm{Fe}_{\mathrm{d}} / \mathrm{Fe}_{\mathrm{o}}$ \\
\hline & \multicolumn{2}{|c|}{ - $\mathrm{mg} \mathrm{kg}^{-1}$} & \multicolumn{2}{|c|}{$-\mathrm{g} \mathrm{kg}^{-1}-$} & \multicolumn{4}{|c|}{$-\mathrm{cmol}_{\mathrm{c}} \mathrm{kg}^{-1}$} & \multicolumn{2}{|c|}{$-\mathrm{g} \mathrm{kg}^{-1}-$} & \\
\hline Planossolo $\mathrm{P}_{\mathrm{SM}}{ }^{(1)}$ & 18,9 & 112 & 210 & 19 & 1,6 & 11,0 & 1,4 & 13,8 & 1,2 & 4,9 & 0,24 \\
\hline Planossolo $\mathrm{P}_{\mathrm{PEL}}$ & 6,8 & 48 & 200 & 18 & 0,7 & 9,2 & 4,4 & 15,1 & 1,2 & 3,9 & 0,30 \\
\hline Chernossolo $\mathrm{C}_{\mathrm{UR}}$ & 10,9 & 132 & 570 & 32 & 0,0 & 11,0 & 6,9 & 23,2 & 8,7 & 13,5 & 0,64 \\
\hline Chernossolo $\mathrm{C}_{\mathrm{SVP}}$ & 25,8 & 224 & 230 & 29 & 0,0 & 12,0 & 3,1 & 19,1 & 0,6 & 0,9 & 0,66 \\
\hline Gleissolo $\mathrm{G}_{\mathrm{SM}}$ & 9,3 & 108 & 290 & 42 & 2,0 & 6,4 & 13,7 & 22,9 & 6,6 & 9,0 & 0,73 \\
\hline Gleissolo $\mathrm{G}_{\mathrm{CA}}$ & 10,1 & 52 & 190 & 11 & 0,1 & 2,1 & 2,5 & 5,5 & 1,6 & 3,5 & 0,47 \\
\hline
\end{tabular}

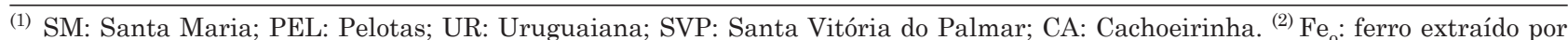
oxalato ácido de amônio; $\mathrm{Fe}_{\mathrm{d}}$ : ferro extraído por ditionito-citrato-bicarbonato. 
Cada solo foi acondicionado em vasos plásticos de 8 L (Figura 1), num total de nove vasos para cada tipo de solo. A quantidade de solo distribuída em cada vaso foi de $4 \mathrm{~kg}$. Os tratamentos foram: testemunha sem adição de $\mathrm{P} ; 100 \mathrm{mg} \mathrm{kg}^{-1}$ de $\mathrm{P}$ total no solo na forma de superfosfato simples (SFS); e $100 \mathrm{mg} \mathrm{kg}^{-1}$ de $\mathrm{P}$ total no solo na forma de superfosfato simples contendo impurezas de fosfato de Fe (SFS + P-Fe) (Quadro 2), conforme análises realizadas no laboratório de fertilizantes da empresa Roullier, localizado em Rio Grande (RS). O experimento foi conduzido em delineamento completamente casualizado com três repetições.

Na montagem dos vasos, instalou-se em cada um deles um sistema de coleta da solução do solo, localizado no centro dos vasos, a $5 \mathrm{~cm}$ de profundidade (Figura 1). Esse sistema objetivou a remoção da solução após o alagamento, para posterior análise de $\mathrm{pH}$, Eh e teores de $\mathrm{P}$ e Fe. O sistema de coleta da solução do solo foi construído a partir de canos de PVC rígido de $0,10 \times 0,025 \mathrm{~m}$, perfurado, recoberto com uma tela de poliamida de $0,80 \mu \mathrm{m}$. No centro do cano foi conectado um segmento de PVC flexível (mangueira de plástico) ligado a um sifão de vidro, em cuja extremidade fora do vaso foi possível a coleta da solução por meio de drenagem livre ou por sucção, quando necessário. Após a montagem dos vasos e aplicação dos tratamentos, os solos foram alagados com água destilada e o nível

Quadro 2. Teores de $\mathrm{P}$ total, solúvel em citrato neutro de amônio (CNA) + água $\left(\mathrm{H}_{2} \mathrm{O}\right)$ e em água das duas fontes de fertilizantes (SFS e SFS + P-Fe)

\begin{tabular}{|c|c|c|c|}
\hline Fonte & P total & $\mathrm{CNA}+\mathrm{H}_{2} \mathrm{O}$ & $\mathrm{H}_{2} \mathrm{O}$ \\
\hline & & $\% \mathrm{P}_{2} \mathrm{O}_{5}$ & 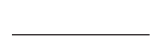 \\
\hline Superfosfato simples (SFS) & 18,7 & $17,5(94 \%)^{(1)}$ & $13,9(79 \%)^{(2)}$ \\
\hline SFS com resíduo (SFS + P-Fe) & 18,2 & $15,8(87 \%)$ & $9,2(58 \%)$ \\
\hline
\end{tabular}

(1) Número entre parênteses representa a percentagem em relação ao $\mathrm{P}$ total. ${ }^{(2)}$ Número entre parênteses representam a percentagem em relação ao $\mathrm{P}$ em $\mathrm{CNA}+\mathrm{H}_{2} \mathrm{O}$. foi mantido a $5 \mathrm{~cm}$ de altura acima do solo durante todo o período de avaliação da sua solução, que se estendeu por 45 dias. Transcorrido o alagamento dos solos, procedeu-se à coleta da solução do solo semanalmente, durante oito semanas, nas seguintes datas: 08/01, 12/01, 16/01, 23/01, 30/01, 06/02, 13/02 e $20 / 02 / 2008$.

A coleta da solução do solo foi feita com uma seringa de $60 \mathrm{~mL}$, implantada no sistema de coleta da solução fixado nos vasos. Após a coleta da solução do solo, procedeu-se imediatamente à avaliação do $\mathrm{pH}$ e do potencial redox por meio de eletrodos específicos (no caso do potencial redox, foi utilizado um eletrodo do tipo $\mathrm{AgCl} / \mathrm{KCl}$ com anel de platina), conectados a um potenciômetro portátil. Para converter as leituras do potencial redox medido em padrão Eh, foi adicionado o valor de $220 \mathrm{mV}$ às leituras. Após a leitura do $\mathrm{pH}$ e Eh, a solução coletada foi armazenada em frascos plásticos de $60 \mathrm{~mL}$, acidificada com $1 \mathrm{~mL}$ de $\mathrm{HCl}$ $1 \mathrm{~mol} \mathrm{~L}^{-1}$ para interromper as reações de oxirredução e, posteriormente, analisada em laboratório. As concentrações de Fe na solução do solo foram determinados utilizando espectrofotômetro de absorção atômica e conforme método descrito por Tedesco et al. (1995), e as de P em colorímetro, segundo método descrito por Murphy \& Riley (1962).

Os valores de $\mathrm{pH}$ e Eh e as concentrações de Fe e P das oito avaliações na solução de cada solo foram submetidos à análise de variância pelo teste $\mathrm{F}$, considerando um esquema fatorial (3 fontes de $\mathrm{P}$ x 8 épocas de coleta); quando significativas, as médias dos tratamentos em cada solo foram comparadas pelo teste de Tukey a $5 \%$. O programa utilizado nas análises estatísticas foi o ASSISTAT (2008).

\section{RESULTADOS E DISCUSSÃO}

Os valores de $\mathrm{pH}$ da solução do solo aumentaram em todos os solos, com exceção do $\mathrm{C}_{\mathrm{SVP}}$, durante os primeiros 10 dias de alagamento (Figura 2), mantendo-se estáveis após esse período, variando entre

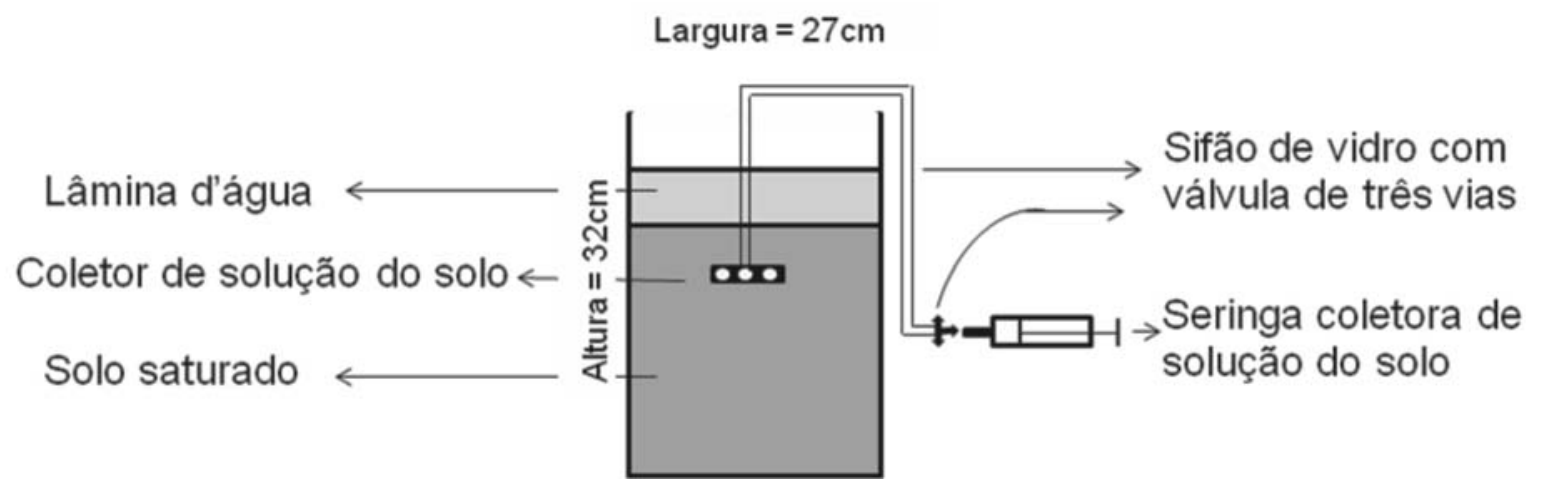

Figura 1. Esquema ilustrando o vaso e o sistema de coleta de solução do solo. 


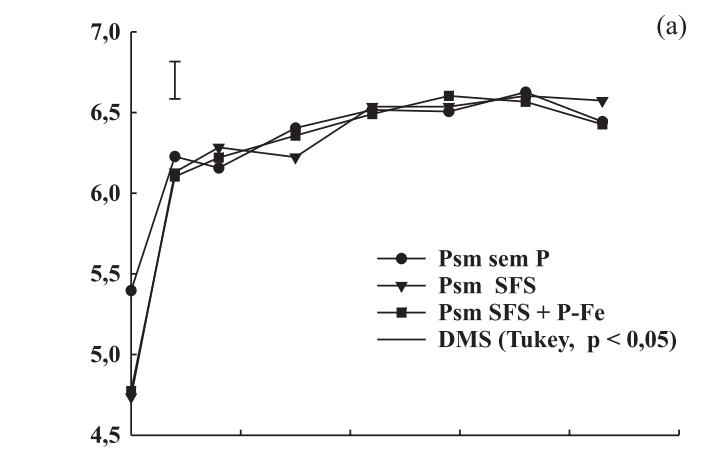

(a)
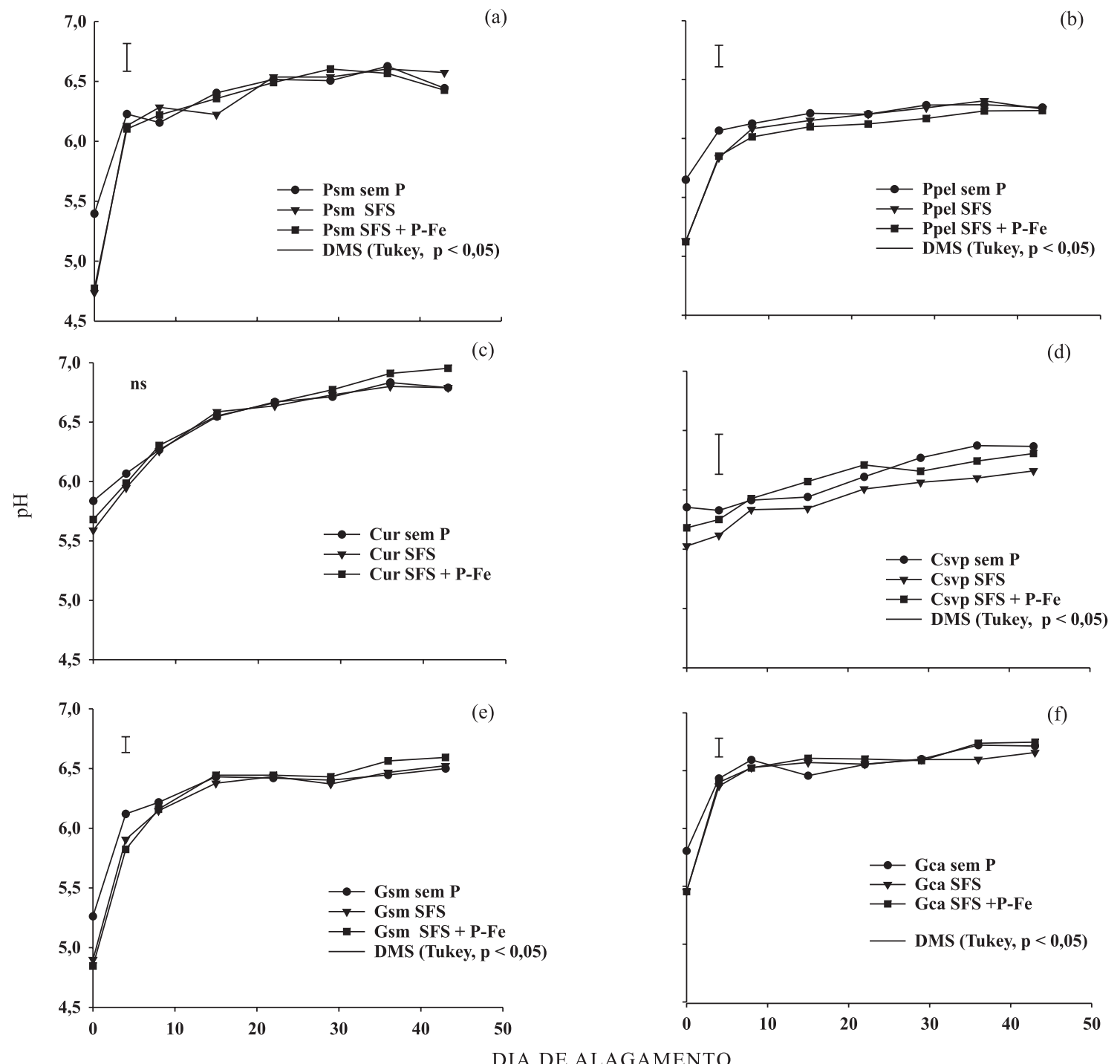

Figura 2. Alterações do valor de pH da solução do solo de seis solos de várzea, submetidos a três fontes de fósforo durante 45 dias. Psm: Planossolo de Santa Maria(a); Ppel: Planossolo de Pelotas (b); Cur: Chernossolo de Uruguaiana (c); Csvp: Chernossolo de Santa Vitória do Palmar (d); Gsm: Gleissolo de Santa Maria (e) Gca: Gleissolo de Cachoeirinha (f). As barras indicam a diferença mínima significativa entre fontes de $\mathbf{P}$ dentro de cada solo pelo teste de Tukey $(p<0,05)$ e ns: variância fator fonte de $P$ não significativa $(p<0,05)$.

6,0 e 6,5 entre os solos. O aumento do pH na solução do solo ocorre pela presença de microrganismos anaeróbios e anaeróbios facultativos nesses ambientes, que, durante o processo respiratório, utilizam como aceptores finais de elétrons compostos oxidados, como óxidos de $\mathrm{Fe}, \mathrm{Mn}$, nitrato e sulfato (Moreira \& Siqueira, 2006; Sousa et al., 2006); essas reações consomem grande quantidade de $\mathrm{H}^{+}$, diminuindo consequentemente o número de prótons de $\mathrm{H}$ na solução do solo e promovendo a elevação do pH (Bartlett \& James, 1993).

Houve interação entre os tratamentos de $\mathrm{P}$ e as épocas de coleta para os solos estudados (exceto para o solo Chernossolo $\mathrm{C}_{\mathrm{UR}}$, em que apenas o fator época de coleta foi significativo). Nesses casos, houve diferença significativa de $\mathrm{pH}$ entre a aplicação de $\mathrm{P}$ e a testemunha sem $\mathrm{P}$, independentemente da fonte aplicada, para a maioria dos solos (Planossolos $\mathrm{P}_{\mathrm{SM}} \mathrm{e}$ $\mathrm{P}_{\mathrm{PEL}}$, Gleissolos $\mathrm{G}_{\mathrm{SM}}$ e $\mathrm{G}_{\mathrm{CA}}$ ) apenas nas duas primeiras coletas ou até aproximadamente os quatro dias de alagamento (Figura 2a,b,e,f). Isso deve ter ocorrido porque, durante o processo de produção do fertilizante, quando não ocorre a completa neutralização do ácido sulfúrico usado na produção do superfosfato simples, gera-se a acidez livre do fertilizante. Assim, diminuição do pH logo após a aplicação de $\mathrm{P}$ deve estar associada com a acidez livre do SFS, independentemente de sua solubilidade. Após 
o oitavo dia de alagamento os valores de $\mathrm{pH}$ não se diferenciaram entre os tratamentos em cada solo, sugerindo a ação do efeito-tampão da acidez dos solos sobre a acidez liberada pelos fertilizantes. Já para o Chernossolo $\mathrm{C}_{\mathrm{SVP}}$ não ocorreu diferença significativa de $\mathrm{pH}$ em nenhuma das coletas, quando comparadas às fontes de $\mathrm{P}$ e à testemunha (Figura 2d). No Chernossolo $\mathrm{C}_{\mathrm{UR}}$ não houve efeito da adição de $\mathrm{P}$ nas oito coletas da solução do solo (Figura 2c). Estudando a solubilização de fosfato natural e fosfato solúvel em dois solos alagados, Gonçalves et al. (2008) observaram que o $\mathrm{pH}$ da solução do solo não diferiu entre as fontes de $\mathrm{P}$ na maior parte do tempo. Esses autores sugerem ainda que isso pode ser importante nesse tipo de estudo, pois, quando não há diferenças significativas nos valores de $\mathrm{pH}$, as possíveis diferenças de solubilidade
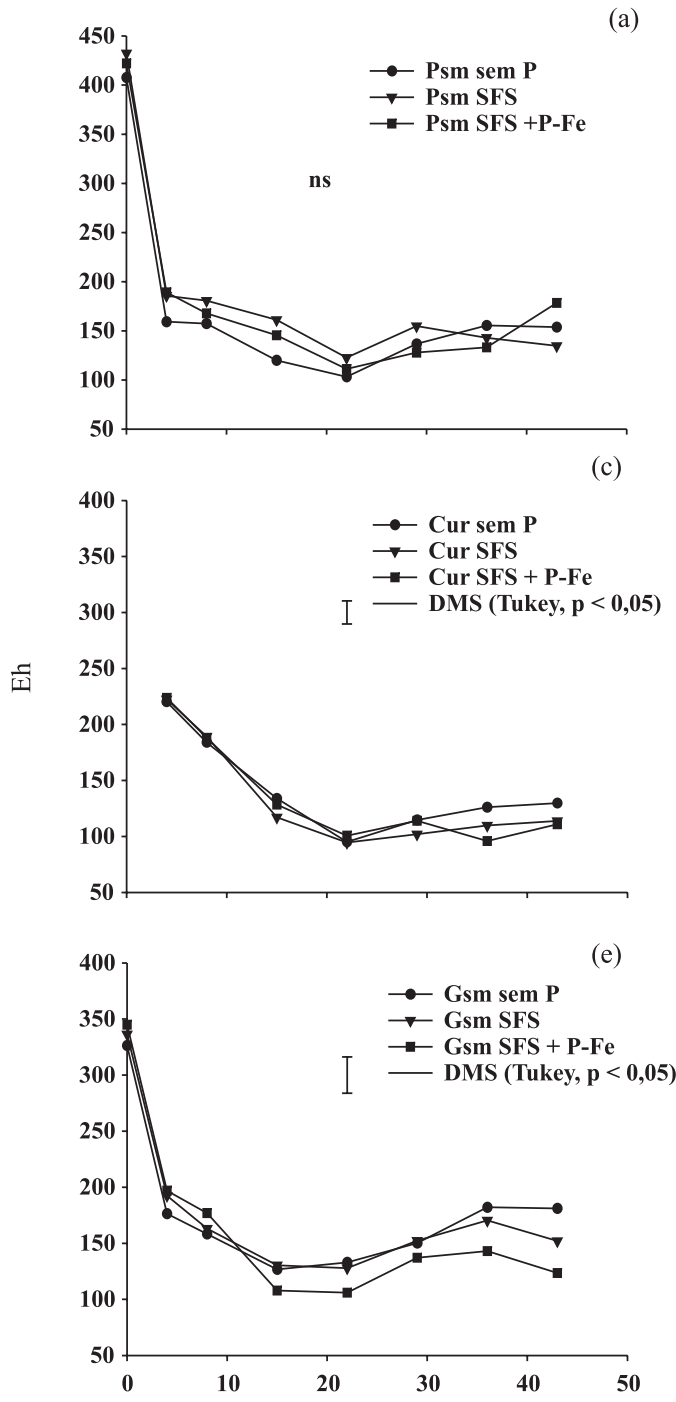

(a)

\section{(c)}

DIA do $\mathrm{P}$ no solo entre as fontes de $\mathrm{P}$ não podem ser atribuídas à influência do $\mathrm{pH}$ sobre a dinâmica do $\mathrm{P}$, e sim às características das fontes.

As reações de redução que ocorrem nos solos consomem íons $\mathrm{H}^{+}$, elevam o $\mathrm{pH}$ e também alteram o Eh do solo, sendo este um atributo para avaliar o grau de redução do solo. Considera-se que, quando o solo apresenta valores de Eh positivos, encontra-se oxidado, e quando apresenta valores negativos, encontra-se reduzido (Camargo et al., 1993). Os valores de Eh na solução do solo aos $5 \mathrm{~cm}$ de profundidade diminuíram em todos os solos devido ao alagamento (Figura 3a,b,c,d,f), passando de valores próximos a $400 \mathrm{mV}$ para valores próximos de $100 \mathrm{mV}$. Em alguns solos alagados o Eh inicial pode ultrapassar $600 \mathrm{mV}$ e reduzir até valores abaixo de zero, devido ao

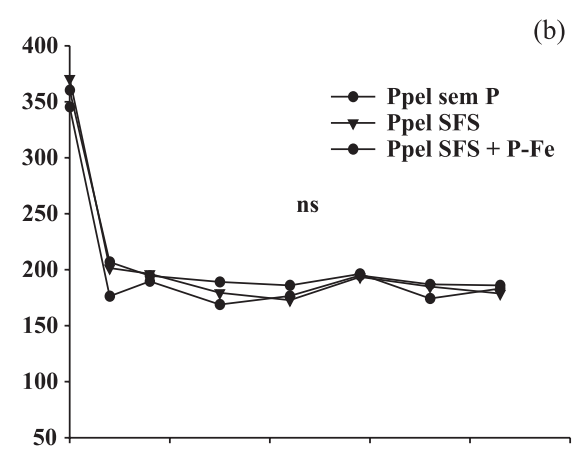

(d)
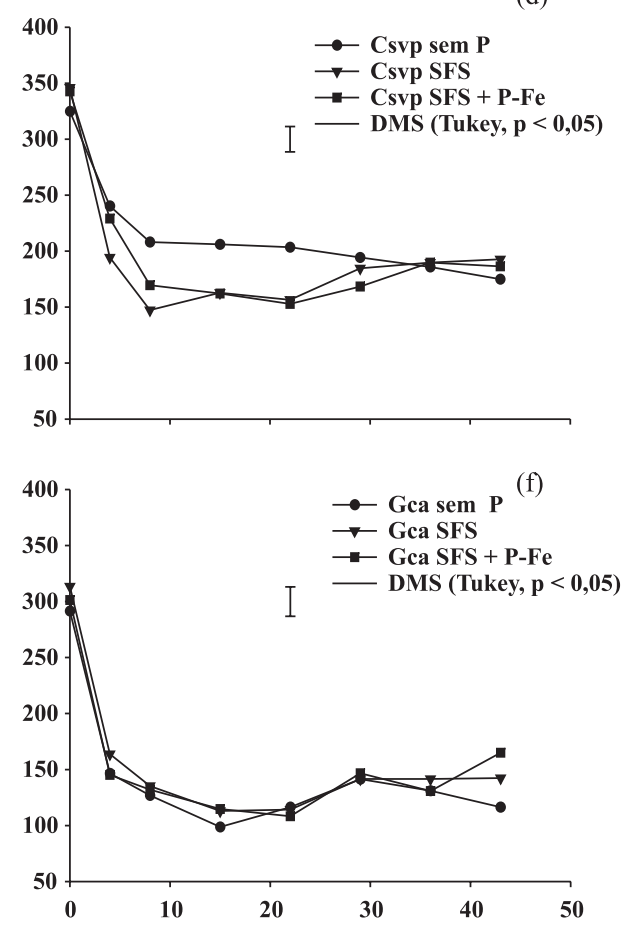

Figura 3. Alterações do valor de Eh da solução do solo de seis solos de várzea submetidos a três fontes de fósforo durante 45 dias. Psm: Planossolo de Santa Maria(a); Ppel: Planossolo de Pelotas (b); Cur: Chernossolo de Uruguaiana (c); Csvp: Chernossolo de Santa Vitória do Palmar (d); Gsm: Gleissolo de Santa Maria (e) Gca: Gleissolo de Cachoeirinha (f). As barras indicam a diferença mínima significativa entre fator $P$ dentro de cada solo pelo teste de Tukey $(p<0,05)$ e ns: variância fator $P$ não significativa $(\mathrm{p}<0,05)$. 
alagamento (Dynia \& Barbosa Filho, 1993), o que depende das características do solo e da profundidade considerada (Sousa, 2001). Essas condições indicam o curso da redução dos solos e, na sequência esperada, que o Fe(III) dos compostos de Fe no solo está sendo reduzido.

Não houve efeito da interação entre as fontes de $\mathrm{P}$ e a época de coleta para os valores de Eh nos Planossolos $\mathrm{P}_{\mathrm{SM}}$ e $\mathrm{P}_{\mathrm{PEL}}$ (Figura 3a,b). Mesmo quando a interação foi significativa, nos Gleissolos $\mathrm{G}_{\mathrm{SM}} \mathrm{e}$ $\mathrm{G}_{\mathrm{CA}}$ (Figura 3e,f), não foi observada diferença significativa de Eh entre as fontes de $\mathrm{P}$ aplicadas em cada época de coleta. O Chernossolo $\mathrm{C}_{\mathrm{UR}}$ (Figura 3c) apresentou diferença significativa de Eh entre a adição de $\mathrm{P}$ e a testemunha somente na primeira coleta. Comportamento distinto ocorreu no Chernossolo $\mathrm{C}_{\mathrm{SVP}}$
(Figura 3d), que mostrou diferença significativa nos valores de Eh entre a aplicação de P (independentemente das fontes utilizadas) e a testemunha durante quase todo o período de alagamento.

As alterações que ocorreram no solo em decorrência do alagamento refletem-se nos valores de $\mathrm{pH}$, Eh e também nos teores de $\mathrm{Fe}^{2+}$ na solução. A diminuição do $\mathrm{O}_{2}$ no solo faz com que bactérias adaptadas para utilizar outros compostos diferentes do $\mathrm{O}_{2}$ os reduzam, promovendo aumento na concentração de formas reduzidas, como evidenciado para os teores de Fe nos seis solos estudados neste trabalho (Figura 4). O aumento do teor de $\mathrm{Fe}^{2+}$ em solos alagados pode ser explicado pela presença de bactérias redutoras capazes de realizar a redução do $\mathrm{Fe}$ (III) a Fe(II), aumentando sua solubilidade (Silva et al., 2003). As maiores
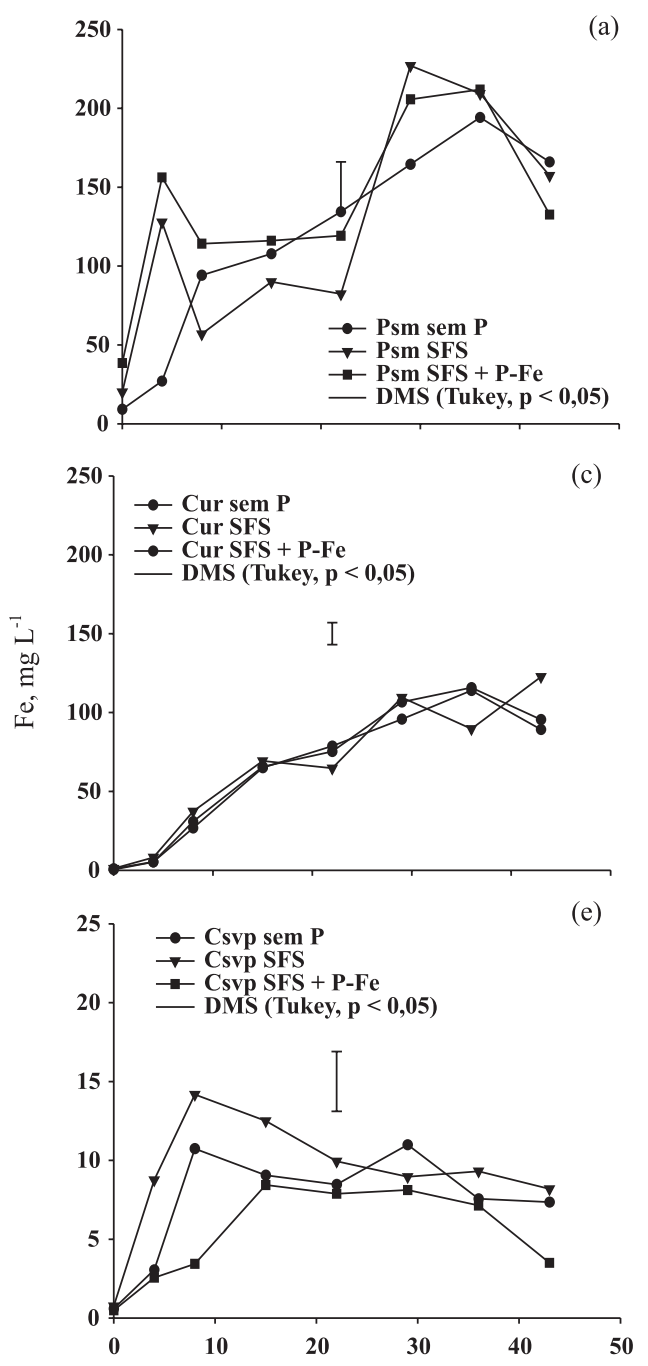
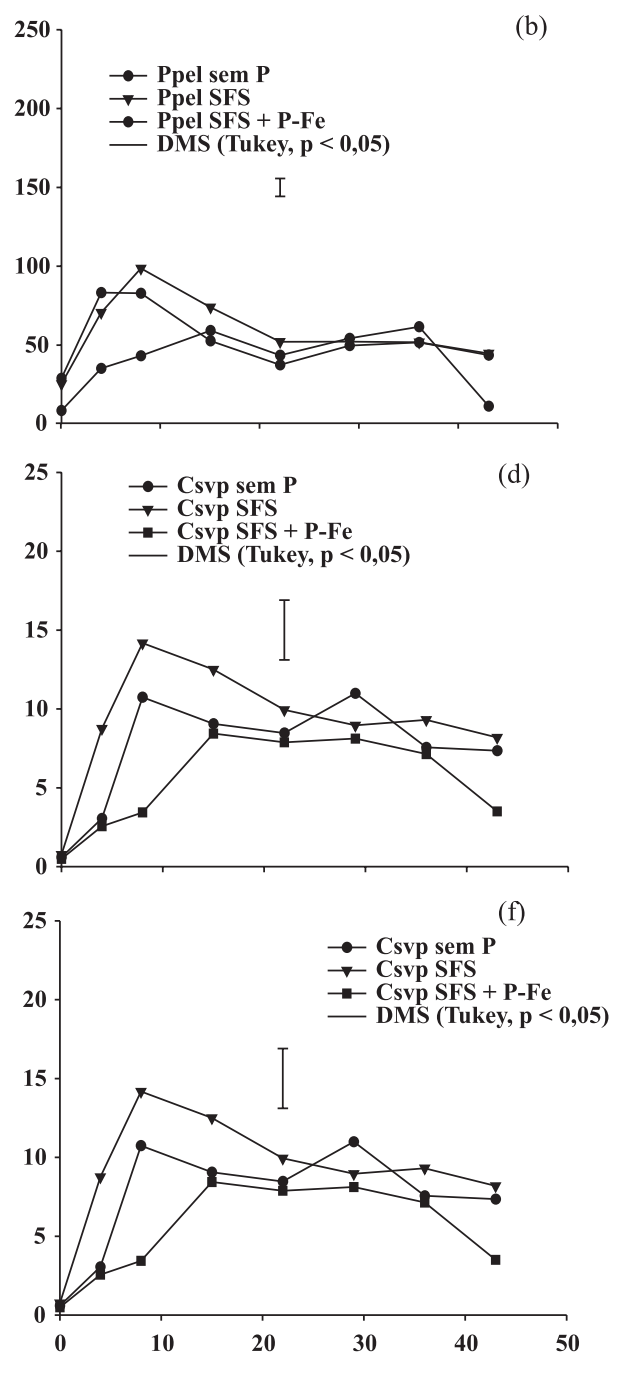

Figura 4. Alterações do teor de Fe da solução do solo de seis solos de várzea submetidos a três fontes de fósforo durante 45 dias. Psm: Planossolo de Santa Maria (a); Ppel: Planossolo de Pelotas (b); Cur: Chernossolo de Uruguaiana (c); Csvp: Cheronossolo de Santa Vitória do Palmar (d); Gsm: Gleissolo de Santa Maria (e) Gca: Gleissolo de Cachoeirinha (f). As barras indicam a diferença mínima significativa entre fontes de P dentro de cada solo pelo teste de Tukey $(p<0,05)$. 
concentrações de Fe na solução do solo (acima de $200 \mathrm{mg} \mathrm{L}^{-1}$ ) foram encontradas no Planossolo $\mathrm{P}_{\mathrm{SM}} \mathrm{e}$ Gleissolo $\mathrm{G}_{\mathrm{SM}}$ (Figura 4), coincidindo justamente com a variação de pH (Figura 2a,e) e Eh (Figura 3a,e). Já os menores valores de Fe na solução ocorreram no Chernossolo $\mathrm{C}_{\mathrm{SVP}}$ e não atingiram $20 \mathrm{mg} \mathrm{L}^{-1}$. A disponibilidade de Fe na solução do solo é influenciada principalmente pelo Eh e também, diretamente, pelo $\mathrm{pH}$, pela qualidade e quantidade de matéria orgânica e pela quantidade e cristalinidade dos óxidos de $\mathrm{Fe}$ (Sousa et al., 2006).

Os Gleissolos $\mathrm{G}_{\mathrm{SM}}$ e $\mathrm{G}_{\mathrm{CA}}$ e o Planossolo $\mathrm{P}_{\mathrm{SM}}$ destacaram-se por apresentar concentrações de Fe na solução significativamente superiores no tratamento com SFS + P-Fe em relação ao SFS comercial, para algumas épocas de coleta. Esses resultados poderiam indicar, num primeiro momento, maior solubilidade desses compostos em ambientes alagados, porém ob- serva-se que o comportamento não foi constante em todos os solos ou coletas, incluindo quando comparado com a testemunha sem $\mathrm{P}$, dificultando uma afirmação segura apenas com esses resultados. O Fe ocorre no solo como óxidos, hidróxidos, oxi-hidróxidos, compostos amorfos, entre outros, e as diferentes formas podem se associar ao P. Com o processo de redução do solo, algumas dessas formas dissociam-se, elevando tanto o teor de Fe na solução como o do elemento ao qual estava ligado. Entretanto, pode haver readsorção do P liberado em outros sítios disponíveis, o que interfe na concentração de P na solução do solo (Gonçalves et al., 2008).

Dessa forma, verificou-se aumento da concentração de $\mathrm{P}$ na solução do solo, mas com variações entre os solos (Figura 5). O P não participa das reações de oxirredução em solos alagados, porém, devido à sua reatividade com os compostos de $\mathrm{Fe}$ passíveis de
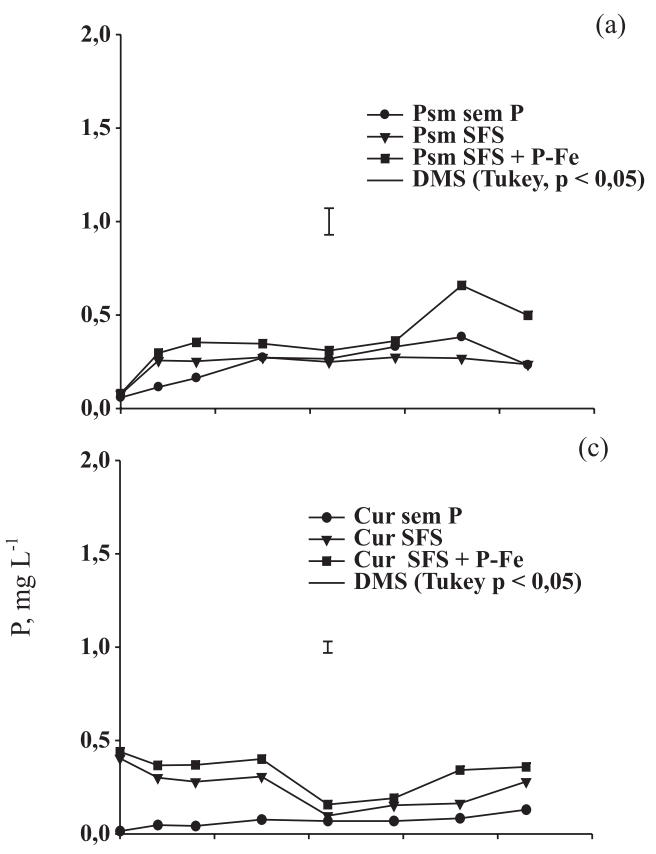

(e)

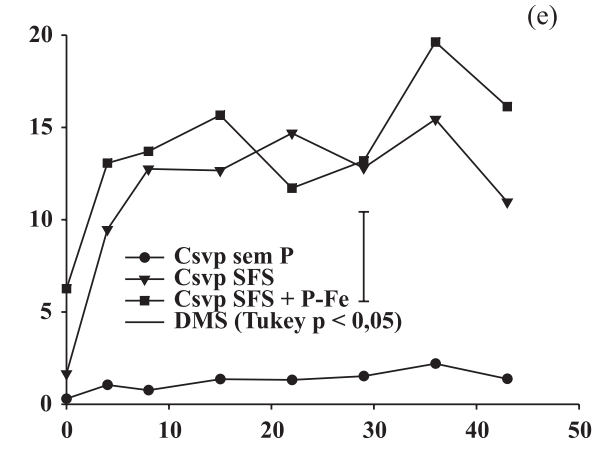

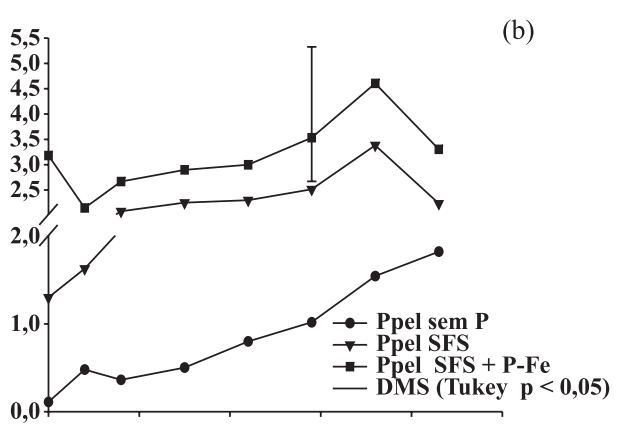

(d)
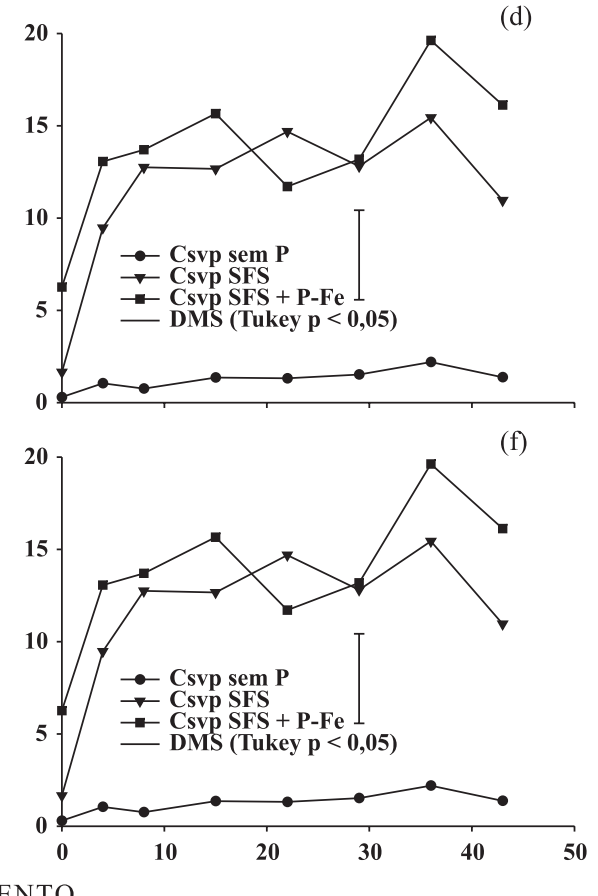

Figura 5. Alterações do teor de fósforo da solução de seis solos de várzea submetidos a três fontes de fósforo durante 45 dias. Psm: Planossolo de Santa Maria(a); Ppel: Planossolo de Pelotas (b); Cur: Chernossolo de Uruguaiana (c); Csvp: Chernossolo de Santa Vitória do Palmar (d); Gsm: Gleissolo de Santa Maria (e) Gca: Gleissolo de Cachoeirinha (f). As barras indicam a diferença mínima significativa entre tratamentos dentro de cada solo pelo teste de Tukey $(p<0,05)$. 
redução, seu comportamento é profundamente alterado pelo curso da redução (Vahl, 2004; Sousa et al., 2006). As menores concentrações de P na solução foram observadas no Planossolo $\mathrm{P}_{\mathrm{SM}}$, Chernossolo $\mathrm{C}_{\mathrm{UR}}$ e no Gleissolo $\mathrm{G}_{\mathrm{SM}}$ (Figura 5a,c,e). O Planossolo $\mathrm{P}_{\mathrm{SM}}$ não apresentou efeito dos tratamentos na maior parte das coletas, havendo diferença entre as fontes de $\mathrm{P}$ apenas nas últimas duas coletas. Fato semelhante pode ser observado no Gleissolo $\mathrm{G}_{\mathrm{SM}}$.

As menores concentrações de $\mathrm{P}$ nesses solos não condizem com os valores de Fe liberados (Figura 4). É possível que outros fatores tenham influenciado a disponibilidade de P. A baixa disponibilidade desse elemento na solução do solo, em alguns solos, é consequência de sua adsorção na fase sólida do solo, sobretudo com os óxidos e hidróxidos de $\mathrm{Fe}$ e $\mathrm{Al}$ (Havlin et al., 2005). Segundo Fox \& Kamprath (1970), a adsorção de P no solo é mais intensa nos solos que contêm maior quantidade de minerais mal cristalizados do que naqueles com maior quantidade de minerais cristalinos. Essas ligações geralmente formam compostos estáveis de alta energia de ligação e baixa solubilidade. De maneira geral, quanto mais argiloso e intemperizado o solo, menores os valores observados de P em solução e maior a capacidade máxima de adsorção; consequentemente, menor a disponibilidade de $\mathrm{P}$ às plantas (Sousa et al., 2006).

A adição de $\mathrm{P}$ elevou destacadamente os teores de $\mathrm{P}$ na solução do solo em todas as coletas no Planossolo $\mathrm{P}_{\mathrm{PEL}}$. Entretanto, mesmo havendo aumento da concentração de P na solução, não foi possível detectar diferença significativa de liberação de $\mathrm{P}$ das fontes quando comparadas à testemunha durante o maior período de alagamento no Planossolo $\mathrm{P}_{\mathrm{PEL}}$, Chernossolo $\mathrm{C}_{\mathrm{SVP}}$ e no Gleissolo $\mathrm{G}_{\mathrm{CA}}$ (Figura 5b,d,f). No caso do Chernossolo $C_{S V P}$, não houve efeito da interação entre tratamentos e a época de coleta, porém houve efeito principal entre as concentrações de P; a adição desse elemento foi significativamente maior que a da testemunha, independentemente da fonte utilizada (Figura 5d). Já o Gleissolo $\mathrm{G}_{\mathrm{CA}}$ foi o único solo em que houve diferença entre as fontes de P: o SFS + P-Fe foi significativamente superior ao SFS nas primeiras coletas (Figura 5f).

Esse comportamento coincidiu com a liberação de Fe para a solução do solo nas primeiras coletas (Figura 4f), o que poderia reforçar a possibilidade de maior solubilidade ao P contido no SFS + P-Fe nessas condições de solo e alagamento. Utilizando compostos (Fe-Al-P) sintetizados semelhantes aos residuais presentes em superfosfatos simples, cujas denominações $\mathrm{H} 8$ e $\mathrm{H} 14$ derivam das fórmulas químicas $\mathrm{Fe}_{3} \mathrm{KH}_{8}\left(\mathrm{PO}_{4}\right)_{6} \cdot 6 \mathrm{H}_{2} \mathrm{O}$ e $\mathrm{Fe}_{3} \mathrm{KH}_{14}\left(\mathrm{PO}_{4}\right)_{8} .4 \mathrm{H}_{2} \mathrm{O}$, respectivamente, Biasoli (2003) concluiu que esses compostos Fe-K-P podem liberar $\mathrm{P}$ com o aumento do $\mathrm{pH}$, o que ocorre com o alagamento do solo. De qualquer modo, os resultados obtidos demonstram que a liberação de $\mathrm{P}$ para a solução em todos os solos utilizados não foi influenciada negativamente pela presença de compostos de baixa solubilidade em água no SFS, o que evidencia o potencial de sua utilização para a cultura do arroz irrigado, conforme os trabalhos de Prochnow (2001, 2003). Mesmo sem atendimento à legislação de fertilizantes em vigor no Brasil, o superfosfato simples contendo impurezas de fosfato de Fe de baixa solubilidade em água tem potencial para uso na cultura do arroz irrigado por alagamento, o que pode representar uma fonte mais barata desse insumo às lavouras de arroz.

\section{CONCLUSÃO}

O superfosfato simples contendo impurezas de fosfato de Fe insolúveis em água, quando aplicado em solos inundados, apresenta comportamento na solução do solo semelhante ao do superfosfato simples sem impurezas.

\section{AGRADECIMENTOS}

Ao professor Rogério Oliveira de Sousa, da UFPel, e aos pesquisadores do Instituto Rio-Grandense do Arroz (IRGA), pela coleta dos solos utilizados. Ao Dr. Nelson Horowitz e à empresa Roullier, pelas análises de $\mathrm{P}$ nos fertilizantes. Ao CNPq, pela concessão de bolsa de Produtividade em Pesquisa e pelo auxílio financeiro ao projeto. À CAPES, pela concessão de bolsa de estudos.

\section{LITERATURA CITADA}

ASSISTAT - Assistência em Estatística Campina Grande, 2008. Versão 7.5 Beta. Disponível em:<http:/www.assistat.com>. Acesso em: 7 de jun. 2008.

BARTLETT, R.J. \& JAMES, B.R. Redox chemistry of soil. Adv. Agron., 50:151-208, 1993.

BIASOLI, G.M. Influência do $\mathrm{pH}$ e da força iônica na liberação de fósforo de compostos do tipo Fe-K-P presentes em superfosfatos. Piracicaba, Escola Superior de Agricultura Luiz de Queiroz, 2003. 76p. (Tese de Mestrado)

BRADY, N.C. \& WEIL, R.R. Nature and properties of soils. 13.ed. New Jersey, Prentice Hall, 2002. 960p.

BRAGA, G. Eficiência de fosfatos com solubilidade variável em água em solos com capacidade de fixação de fósforo induzida. Piracicaba, Escola Superior de Agricultura Luiz de Queiroz, 2006. 82p. (Tese de Mestrado)

BRASIL. Decreto n. 4954 de 14 de janeiro de 2004, anexo II. Aprova o Regulamento da Lei $\mathrm{n}^{\circ} 6.894$, de 16 de dezembro de 1980, que dispõe sobre a inspeção e fiscalização da produção e do comércio de fertilizantes, corretivos, inoculantes ou biofertilizantes destinados à agricultura, e dá outras providências. Brasília, 2004. 32p. 
CAMARGO, F.A.O.; SANTOS, G.A. \& ROSSIELO, R.O.P. Efeito dos ácidos acético e butírico sobre o crescimento de plântulas de arroz. Pesq. Agropec. Bras., 28:1011-1018, 1993.

DYNIA, J.F. \& BARBOSA FILHO, M.P. Alterações de pH, Eh e disponibilidade de micronutrientes para o arroz irrigado em um solo de várzea tratado com calcário e palha de arroz em casa de vegetação. R. Bras.Ci. Solo, 17:67-74, 1993.

FOX, R.L. \& KAMPRATH, E.J. Phosphate sorption isotherms for evaluating the phosphate requirements of soil. Soil Sci. Soc. Am. J., 34:902-907, 1970.

GONÇALVES, G.K.; SOUSA, R.O.; VAHL, L.C. \& BORTOLON, L. Solubilização dos fosfatos naturais Patos de Minas e Arad em dois solos alagados. R. Bras.Ci. Solo, 32: 2157-2164, 2008.

HAVLIN, J.L.; TISDALE, S.L.; BEATON, J.D.; \& NELSON, W.L. Soil fertility and fertilizers: An introduction to nutrient management. 7.ed. New Jersey, Prentice Hall, 2005. 515 p.

KAMINSK, J. \& PERUZZO, G. Eficácia dos fosfatos naturais reativos em sistemas de cultivo. Santa Maria, Núcleo Regional Sul/Sociedade Brasileira de Ciência do Solo, 1997. 31p. (Boletim Técnico, 3).

MOREIRA, F.M.S. \& SIQUEIRA, J.O. Microbiologia e bioquímica do solo. 2.ed. Lavras, Universidade Federal de Lavras, 2006. 729p.

MURPHY, J. \& RILEY, J.P. A modified single solution method for the determination of phosphate in natural waters. Anal. Chim. Acta, 27:31-36, 1962.

PONNAMPERUMA, F.N. The chemistry of submerged soils. Adv. Agron., 24:29-96, 1972

PROCHNOW, L.I. Eficiência agronômica de fosfatos acidulados contendo compostos fosfáticos de ferro para o arroz de sequeiro e arroz inundado. Piracicaba, Escola Superior de Agricultura Luiz de Queiroz, 2001. 118p. (Tese de Livre Docência)

PROCHNOW, L.I.; CHIEN, S.H.; DILLARD, E.F.; AUSTIN, E.R.; CARMONA, G.; HENAO, J.; SINGH, U. \& TAYLOR, R.W. Synthesis, characterization, and agronomic evaluation of iron phosphate impurities in superphosphates. Soil Sci. Soc.Am. J., 67:1551-1563, 2003.
PROCHNOW, L.I.; QUISPE, J.F.S.; FRANCISCO, E.A.B. \& BRAGA, G. Effectiveness of phosphate fertilizers of different water solubilities in relation to soil phosphorus adsorption. Sci. Agric., 63:333-340, 2006.

RANNO, S.K. Estimativa da disponibilidade de fósforo para a cultura do arroz irrigado em solos do RS. Santa Maria, Universidade Federal de Santa Maria, 2004. 139p. (Tese de Mestrado)

RANNO, S.K.; SILVA, L.S.; GATIBONI, L.C. \& RHODEN, A. Capacidade de adsorção de fósforo em solos de várzea do Estado do Rio Grande do Sul. R. Bras. Ci. Solo, 31:21-28, 2007.

SILVA, L.S.; SOUSA, R.O. \& BOHNEN, H. Alterações no teores de nutrientes em dois solos alagados, com e sem plantas de arroz. Ci. Rural, 33:487-490, 2003.

SOUSA, R.O.; CAMARGO, F.A.O. \& VAHL, L.C. Solos alagados (Reações de Redox). In: MEURER, E.J., org. Fundamentos de química do solo. 3.ed. Porto Alegre, Evangraf, 2006. p.185-211.

SOUSA, R.O. Oxirredução em solos alagados afetada por resíduos vegetais. Porto Alegre, Universidade Federal do Rio Grande do Sul, 2001. 180p. (Tese de Doutorado)

SOUSA, R.O.; CAMARGO, F.A. O. \& VAHL, L.C. Solos alagados reações redox. In: MEURER, J.E. Fundamentos de química do solo. 3.ed. Porto Alegre, Evangraf, 2006. p.185-210.

TEDESCO, M.J.; GIANELLO, C.; BISSANI, C.A.; BOHNEN, H. \& VOLKWEISS, S.J. Análise de solo, plantas e outros materiais. 2.ed. Porto Alegre, Universidade Federal do Rio Grande do Sul, 1995. 147p. (Boletim Técnico, 5)

VAHL, L.C. O fósforo na cultura do arroz irrigado. In: YAMADA, T. \& ABDALLA, S.R.S., eds. Fósforo na agricultura brasileira. Piracicaba, Associação Brasileira para a Pesquisa da Potassa e do Fosfato, 2004. p.419-433.

VELlOSO, A.C.X.; OLIVEIRA, C. \& LEAL, J.R. Processos redox em Glei Húmico do Estado do Rio de Janeiro: I. Variação das concentrações de Fe (II) e fosfato. R. Bras. Ci. Solo, 17:27-34, 1993. 